\title{
Anthropometric Method for Determining "Masked Obesity" in the Young Japanese Female Population
}

\author{
Yoshiyuki Fukuoka, ${ }^{1,2}$ Hatsumi Ueoka, ${ }^{1}$ Nahoko Koya, ${ }^{2}$ \\ Yoshihiko Fujisawa, ${ }^{2}$ and Masaru Ishii ${ }^{3}$ \\ ${ }^{1}$ Laboratory of Environmental and Applied Physiology, Faculty of Environmental and Symbiotic Sciences, \\ Prefectural University of Kumamoto, Kumamoto 862-8502, Japan \\ ${ }^{2}$ Faculty of Health and Sports Science, Doshisha University, 1-3 Kyotanabe, Kyoto 610-0394, Japan \\ ${ }^{3}$ Research Center of Physical Culture, Fukuoka University of Education, Fukuoka 811-4192, Japan \\ Correspondence should be addressed to Yoshiyuki Fukuoka, yfukuoka@mail.doshisha.ac.jp \\ Received 10 November 2011; Revised 10 January 2012; Accepted 7 March 2012 \\ Academic Editor: Maryna Steyn
}

Copyright (๑) 2012 Yoshiyuki Fukuoka et al. This is an open access article distributed under the Creative Commons Attribution License, which permits unrestricted use, distribution, and reproduction in any medium, provided the original work is properly cited.

A specific kind of obesity that occurs in young women who appear thin but who have relatively greater percentage of body fat than is normal for their weight is called "masked obesity." The definition of masked obesity is nutritionally and anthropometrically clear: body mass index $(\mathrm{BMI})<25 \mathrm{~kg} / \mathrm{m}^{2}$ and percentage of body fat $(\% \mathrm{BF}) \geq 30 \%$. However, this definition allowed obesity to be underestimated. Thus, we determined that when mathematically analyzing the relationship between BMI and \%BF, the equation $\% \mathrm{BF}=a \mathrm{BMI}^{\mathrm{b}}$ could be applied, where $a$ (i.e., $\% \mathrm{BF} / \mathrm{BMI}^{\mathrm{b}}$ ), converted by logarithmic function, is defined as an index for masked obesity (MOI). The MOI equation (a) was thus $\% \mathrm{BF} / \mathrm{BMI}^{2.12}$. We measured the height, weight, and \%BF of 8,068 young women from all over Japan. The percentage of subjects with masked obesity above the defined criteria (BMI $<25 \mathrm{~kg} / \mathrm{m}^{2}$ and $\left.\% \mathrm{BF} \geq 30 \%\right)$ was $7.4 \%$. The mean MOI was 0.0444 in the masked obesity group and we determined additional cases of masked obesity if the MOI was greater than 0.0444 . The percentage of additional masked obesity cases within the standard scale was $10.4 \%$, and thus, the percentage of masked obesity was $17.8 \%$ in the young Japanese women tested.

\section{Introduction}

The change in eating habits in modern society has led to an increase in the consumption of highly refined foods and Japanese young women in particular are at risk for weight change. Based on a Japanese nutritional survey, body mass index $\left(\mathrm{BMI}=\right.$ weight $/$ height $^{2}$ expressed as $\left.\mathrm{kg} / \mathrm{m}^{2}\right)$ gradually decreased from 21.5 in 1960 to 20.5 in 1995 in women aged 15-24 years [1]. And Tanaka et al. [2] reported that women in 1994-1995 had a significantly lower fat-free mass index $\left(\right.$ FFMI $=$ fat-free mass $/$ height ${ }^{2}$ expressed as $\mathrm{kg} / \mathrm{m}^{2}$ ) than those in 1986 despite a fat-mass index $(F M I=$ fat mass $/$ height $^{2}$ expressed as $\mathrm{kg} / \mathrm{m}^{2}$ ) similar to that of 1986 .

Since a close relationship between BMI and percentage of body fat $(\% \mathrm{BF})$ can be observed, BMI is the most convenient measure for estimating obesity in a wide range of populations. However, a specific kind of obesity that occurs in young women who appear thin but who have a relatively greater $\% \mathrm{BF}$ than is normal for their weight is called "masked obesity." The definition of masked obesity is nutritionally and anthropometrically specific: BMI $<25 \mathrm{~kg} / \mathrm{m}^{2}$ and $\% \mathrm{BF}$ $\geq 30 \%$ (light blue area in Figure 1). In our previous study, we examined young Japanese women at one college who appear thin but have relatively more body fat than is normal for their weight and determined that the percentage of masked obesity was $21.8 \%(84 / 385)$ in that population [3]. Yamagishi et al. [4] also found that 94 of 605 subjects (15.5\%) had a high \%BF, but they did not distinguish between high $\mathrm{BF} \%$ and masked obesity. Our hypothesis was that masked obesity would be present in approximately $20 \%$ of young Japanese woman, if measured by our new approach of using a masked obesity index (MOI) [3]. To our knowledge, a national survey of masked obesity has not yet been conducted in Japan. Therefore, we used a mathematical 


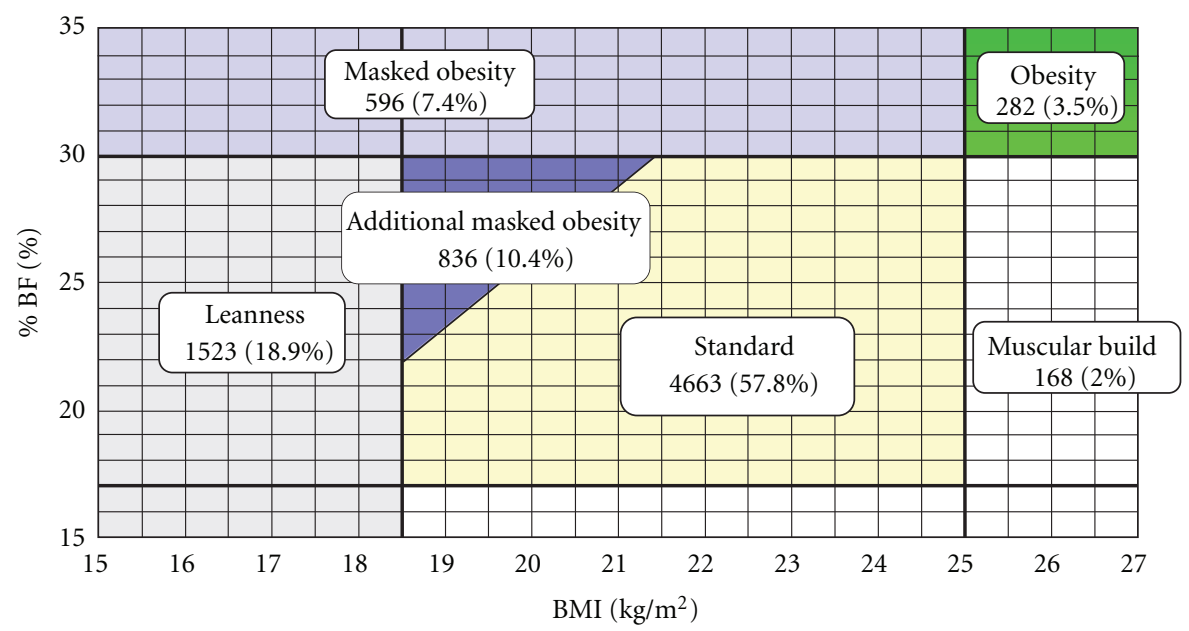

FIGURE 1: Region setting of fundamental categories of obesity, masked obesity, additional masked obesity, standard, muscular build, and leanness, and the distribution diagram in 8,068 young Japanese women.

approach to establish an estimation of masked obesity in the young Japanese woman population.

Japanese women's magazines and morning TV shows frequently report on issues of diets and/or exercise for obtaining a more slender body, and many young women believe what they see and read about how their ideal bodies should look. It may be an implanted idea of what is beautiful that is causing young women to so strongly desire slender bodies like those of fashion models [5, 6]. A woman's perception of her ideal weight is more influenced by cultural standards than by medical implications [5], and women who adhere to a cultural rather than medical standard of ideal weight have three times the risk of developing eating disorders such as anorexia or bulimia [6]. Thus, our second aim was to investigate the actual state of leanness (BMI $\leq 18.5 \mathrm{~kg} / \mathrm{m}^{2}$ and $\left.\% \mathrm{BF}<30 \%\right)$ in the young Japanese population.

\section{Materials and Methods}

2.1. Participants. The subject population consisted of 8,068 , 18-to 21-year-old female students in tertiary institutions and universities throughout Japan. The study was conducted in the period 2000 to 2005, and the subjects were informed of the study protocol prior to enrollment. All study protocols were approved by the ethics committee of each of the participating institutions, and approved consent forms were signed by all individuals prior to enrollment. The enrollment procedure involved anthropometric data for age, weight, height, and percentage of body fat $(\% \mathrm{BF})$, the lifestyle evaluation questionnaire, blood pressure, and 12-lead electrocardiogram. All subjects provided written consent for their participation after they were fully informed about the study. The investigation was performed in compliance with the Declaration of Helsinki.

Percentage of body fat (\%BF) was measured by bioelectrical impedance, hydrostatic weighing, and skinfold measurement. The equipment could not be standardized among the many different institutes involved in our study. However, the principle of the measurements is acceptable.

2.2. Categories in Leanness, Standard Obesity, Masked Obesity, and New Masked Obesity. Leanness is defined as BMI < $18.5 \mathrm{~kg} / \mathrm{m}^{2}$ and $\% \mathrm{BF}<30 \%$. The definition of masked obesity, from a nutritional and anthropometrical stance, is BMI $<25 \mathrm{~kg} / \mathrm{m}^{2}$ and $\% \mathrm{BF} \geq 30 \%$. Obesity is defined as $\mathrm{BMI} \geq 25 \mathrm{~kg} / \mathrm{m}^{2}$ and $\% \mathrm{BF} \geq 30 \%$. However, based on these definitions, masked obesity is likely to be underestimated [3]. We therefore searched for masked obesity within the standard scale of $18.5-25 \mathrm{~kg} / \mathrm{m}^{2}$ BMI and $17-30 \% \% \mathrm{BF}$ (Table 1). Besides these categories, there is the rest that is characterized as muscular build (white area in Figure 1).

To do so, an anthropometric method was developed for evaluating masked obesity using a "masked obesity index" (MOI).

2.3. Model of Mathematical Analysis. We know experientially that there is a curved linear relationship between BMI and $\% \mathrm{BF}$ in the young woman population [3]. When creating a model of mathematical analysis of the relationship between $\mathrm{BMI}$ and $\% \mathrm{BF}$, the equation $\% \mathrm{BF}=a \mathrm{BMI}^{b}$ can be applied [3]. When the logarithm of both scales is used, the equation becomes

$$
\log \% \mathrm{BF}=\log a+b \log \mathrm{BMI}
$$

When individual \%BF and $\mathrm{BMI}$ are substituted in the regression, $\log a$ is directly dependent on the deviation from the regression line. The value $a$ (i.e., $\% \mathrm{BF} / \mathrm{BMI}^{b}$ ), which is converted by logarithmic function, is defined as the MOI. Thus, this MOI can be used to estimate masked obesity using a single variable. To calculate the MOI in the present study, the subjects were classified into five groups based on \%BF (under $10 \%, 10-15 \%, 15-20 \%, 20-25 \%$, and higher than $25 \%$ body fat), and the average BMI and \%BF were then fitted to a regression line in both logarithmic scales. 
TABLE 1: The fundamental categories for obesity, masked obesity, standard, and leanness [3].

\begin{tabular}{lccc}
\hline Category & $\% \mathrm{BF}$ & & $\mathrm{BMI}$ \\
\hline Obesity & $\% \mathrm{BF} \geq 30 \%$ & $\mathrm{An}$ & $\mathrm{BMI} \geq 25 \mathrm{~kg} / \mathrm{m}^{2}$ \\
Maskedobesity & $\% \mathrm{BF} \geq 30 \%$ & And & $\mathrm{BMI}<25 \mathrm{~kg} / \mathrm{m}^{2}$ \\
Standard & $17 \%<\% \mathrm{BF}<30 \%$ & And & $18.5 \mathrm{~kg} / \mathrm{m}^{2}<\mathrm{BMI}<25 \mathrm{~kg} / \mathrm{m}^{2}$ \\
Leanness & $\% \mathrm{BF}<30 \%$ & And & $\mathrm{BMI} \leq 18.5 \mathrm{~kg} / \mathrm{m}^{2}$ \\
\hline
\end{tabular}

BMI: body mass index (wieght $/$ height ${ }^{2}, \mathrm{~kg} / \mathrm{m}^{2}$ )

\%BF: the percentage of body fat using by bioelectrical impedance, hydrostatic weighing, and skinfold measurement.

\section{Results}

The subjects' ages, body weight, and height were $19.1 \pm$ 1.4 yrs (mean \pm standard deviation: $(\mathrm{SD})), 51.3 \pm 6.8 \mathrm{~kg}$, and $158.3 \pm 5.1 \mathrm{~cm}$, respectively. These data are similar to other observations in young Japanese women [1-4]. Judging from the criteria shown in Table 1, the percentage of subjects in the obesity group was $3.5 \%(282 / 8,068$, green area in Figure 1) and that of subjects in the masked obesity group was $7.4 \%$ $(596 / 8,068$, light blue area in Figure 1).

The regression coefficient was calculated as $2.12(r=$ 0.99 ); the MOI equation ( $a$ ) was thus $\% \mathrm{BF} / \mathrm{BMI}^{2.12}$. Using this equation, the MOI values in the masked obesity group ( $n=596)$ were calculated, and the distribution of MOI was localized to the small range of 0.02 between 0.0300 and 0.0499 , which included $91.1 \%$ of the subjects. As a result, the mean MOI value in the masked obesity group was 0.0444 , suggesting that values higher than 0.0444 may be indicative of masked obesity within the standard scale. Thus, the equation was determined as $y=0.0444 \cdot \mathrm{BMI}^{2.12}$. We chose to classify subjects with MOIs higher than the critical value of 0.0444 as being in the additional masked obesity group within the standard scale $\left(18.5 \mathrm{~kg} / \mathrm{m}^{2}<\mathrm{BMI}<\right.$ $25 \mathrm{~kg} / \mathrm{m}^{2}$ and $\left.17 \%<\% \mathrm{BF}<30 \%\right)$. Based on the new baseline of $0.0444 \mathrm{MOI}$, the percentage of those with masked obesity within the standard scale was $10.4 \%(836 / 8,068$; subjects designated as having "additional masked obesity," dark blue area in Figure 1). Consequently, the estimated total $\%$ of the young Japanese woman population with masked obesity was $17.8 \%(10.4 \%$ plus $7.4 \%)$.

The percentage of the standard scale was $57.8 \%$ $(4,663 / 8,068)$ in total, which suggests that the standard scale as a whole falls slightly below $60 \%$ (yellow area in Figure 1). With respect to leanness (Table 1), the percentage of those characterized by leanness, or a BMI below $18.5 \mathrm{~kg} / \mathrm{m}^{2}$, was $18.9 \%(1,523 / 8,068$, grey area in Figure 1$)$ in total. Two percent of the subjects $(168 / 8068)$ were characterized as women who have a muscular build (white areas in Figure 1).

\section{Discussion}

Using this mathematical and anthropometric technique, we might have established an estimation of masked obesity. The percentage of those with masked obesity within the standard scale was $10.4 \%$, and the total $\%$ of masked obesity in the young Japanese woman population was $17.8 \%$ (10.4\% plus $7.4 \%$ ). It is not yet known whether masked obesity will in fact become a serious problem in terms of its epidemiological, biochemical, and pathophysiological issues.

An additional issue concerning the desire to be lean was also detected in the present study because the percentage of those characterized by leanness was $18.9 \%$. Recently, it was reported that excessive attempts to achieve slimness lead to gynecological disorders such as anemia [7].

Based on the National Nutrition Survey of Japan (JNNS), the mean BMI has decreased slightly in women, by $-0.09 \mathrm{~kg} / \mathrm{m}^{2} / 10$ years [8]. Interestingly, Yamagishi et al. [4] focused on the distribution of high \%BF subjects within the whole range of BMI values and found a percentage of $15.5 \%(94 / 605)$, of which the distribution in the normal BMI range from $<18.5$ to $\geq 22.9 \mathrm{~kg} / \mathrm{m}^{2}$ with high $\%$ BF was $7.3 \%$ (44/605). This $7.3 \%$ distribution in the normal BMI range with high $\% \mathrm{BF}$ was consistent with the masked obesity group (7.4\%) (Figure 1).

Recently, perceptions of overweight status and attempts to lose weight were found to be highest in women from the Pacific Asian countries compared to those from northwestern Europe and the USA, central Eastern Europe, the Mediterranean, and South America, indicating that local cultural factors may moderate attitudes toward weight [9]. Japanese women had the highest prevalence of perceived overweight (63\%), with Thailand second in the world [9]. Thus, we think that Asian women are very sensitive to changes in their body weight regardless of the balance of their body composition. Figure 1 can be used to determine the category to which a given young woman belongs.

Using an endocrinological approach, Karelis et al. [10] introduce the endocrinological concept that in comparison to metabolically normal normal-weight subjects, metabolically obese normal-weight (MONW) subjects have higher plasma triglycerides, high visceral fat, and lower high-density lipoprotein (HDL) cholesterol, which is associated with insulin resistance. The MONW group is also characterized by lower physical activity and energy expenditure, which leads to accumulating fat mass without the alteration of body weight.

Recently, the associations between dietary patterns and obesity among young Japanese women were investigated and Japanese traditional food patterns and Western food patterns were both found to be significantly related to an increased risk of obesity even among relatively lean young Japanese women [11]. Furthermore, following healthy food patterns only allowed subjects to achieve a lower risk of obesity. These findings suggest a higher possibility of masked obesity 
within the standard scale between BMI and \%BF in the young Japanese woman population. Further investigations are needed to identify the presence of health risk behaviors in women with masked obesity.

The perception that "I never put on weight no matter what I do" may correctly reflect a stable weight history, rather than being a biological determinant of weight [12]. Thus, intervention strategies aimed at reducing weight gain and obesity in women may also need to focus on psychological factors.

An additional issue concerning the desire to be lean was also detected in the present study, because the percentage of those characterized by leanness was high, at $18.9 \%$. Takimoto et al. [13] observed that the prevalence of thin women (BMI $\left.<18.5 \mathrm{~kg} / \mathrm{m}^{2}\right)$ and extremely thin women $\left(\mathrm{BMI}<17 \mathrm{~kg} / \mathrm{m}^{2}\right)$ has increased significantly over a 25 -year period among 15 to-19-year olds. The Japan Ministry of Health, Labour and Welfare (HLW) research group released a report based on the 2002 J-NNS showing that the percentage of women defined as lean had more than doubled for those in their $20 \mathrm{~s}$, from $11.4 \%$ in 1982 to $26.0 \%$ in 2002 , as well as for those in their $30 \mathrm{~s}$, from $8.1 \%$ in 1982 to $15.1 \%$ in 2002 (Japan Ministry of HLW, 2003) [14]. The same report noted that about $75 \%$ of the young Japanese woman population said that they wanted leaner bodies, and $71 \%$ of women in their $20 \mathrm{~s}$ believed that they tended to be obese, despite the fact that they had a standard body composition (within the standard scale of $18.5-25 \mathrm{~kg} / \mathrm{m}^{2}$ BMI and $17-30 \% \%$ BF, [3, 7]). Thus, it can be stated that a great number of Japanese women want to achieve a degree of slimness that may not be appropriate for their body composition (i.e., the balance between lean body mass and body fat mass) [15].

Body dissatisfaction was found to explain $29 \%$ of the symptomatology of eating disorder in a sample of normal college women [16]. Kuboki et al. [17] report that the incidences of eating disorders among the 13-to-29 year-old Japanese woman population are 20.4 to 25.3 per $10^{5}$ for anorexia and 12.8 to 13.6 per $10^{5}$ for bulimia by analyzing the 1992 data, which are extremely high rates compared to those of other age groups. In addition, women who adhere to a cultural rather than a medical standard of ideal weight have three times the risk of developing eating disorders such as anorexia or bulimia [6]. If we are to develop effective strategies to prevent masked obesity and excessive slenderness in the young Japanese woman population, it is important to develop a better understanding of this population's eating and physical activity behaviors [18].

The limitations of the present study include the following: the participants were all college-educated women, and thus their results may not necessarily be generalized to a wider range of populations of women. Although these data of $\% \mathrm{BF}$ were directly measured, there are methodological differences in bioelectrical impedance, hydrostatic weighing, and skinfold measurement. These three representative measurements are currently widely used in Japan as a method to estimate \%BF in more diverse populations.

In conclusion, using a mathematical and anthropometric method, we established a possible estimation of masked obesity. The masked obesity is a specific type of obesity present in certain young Japanese women. The percentage of those with masked obesity in the young Japanese woman population was $17.8 \%$ (10.4\% as determined by our method plus $7.4 \%$ as determined by the standard method). An additional issue concerning the desire to be lean was also detected in the present study, as reflected by the high percentage of subjects characterized by leanness (18.9\%). These findings suggested that Japanese young women who make excessive attempts to achieve slimness may achieve masked obesity $(17.8 \%)$ or leanness $(18.9 \%)$.

\section{Acknowledgments}

This study was supported in part by Meiji-Yasuda Warfare Company Grant (2003) and Grants-in-Aid for Science Research from the Ministry of Education and Science of Japan (no. 16657076). The authors gratefully acknowledge the expert technical assistance of Miss Aki Sudoh.

\section{References}

[1] N. Kiriike, T. Nagata, K. Sirata, and N. Yamamoto, "Are young women in Japan at high risk for eating disorders?: decreased BMI in young females from 1960 to 1995," Psychiatry and Clinical Neurosciences, vol. 52, no. 3, pp. 279-281, 1998.

[2] S. M. Tanaka, K. Hattori, H. Tobe, T. Satake, and W. C. Chumlea, "Change of body composition over an eight year period among Japanese university students," The Journal of Nutrition, Health \& Aging, vol. 3, pp. 165-168, 1999.

[3] R. Takahashi, M. Ishii, and Y. Fukuoka, "A method for evaluating the masked obesity in young females," Japan Society of Physical Anthropology, vol. 7, pp. 213-217, 2002 (Japanese).

[4] H. Yamagishi, T. Kitano, T. Kuchiki, H. Okazaki, and S. Shibata, "Association between body composition and body mass index in young Japanese women," Journal of Nutritional Science and Vitaminology, vol. 48, no. 3, pp. 201-206, 2002.

[5] S. S. Komia and T. Nakao, Shintaisoseigaku, Gihoudou Press, 2002.

[6] S. Hesse-Biber, Am I Thin Enough yet? Oxford University Press, Oxford, UK, 1996.

[7] T. Liabsuetrakul, "Is international or Asian criteria-based body mass index associated with maternal anaemia, low birthweight, and preterm Births among Thai population?-an observational study," Journal of Health, Population, and Nutrition, vol. 29, pp. 218-228, 2011.

[8] N. Yoshiike, F. Seino, S. Tajima et al., "Twenty-year changes in the prevalence of overweight in Japanese adults: the National Nutrition Survey 1976-95," Obesity Reviews, vol. 3, no. 3, pp. 183-190, 2002.

[9] J. Wardle, A. M. Haase, and A. Steptoe, "Body image and weight control in young adults: international comparisons in university students from 22 countries," International Journal of Obesity, vol. 30, no. 4, pp. 644-651, 2006.

[10] A. D. Karelis, D. H. St-Pierre, F. Conus, R. Rabasa-Lhoret, and E. T. Poehlman, "Metabolic and body composition factors in subgroups of obesity: what do we know?" Journal of Clinical Endocrinology and Metabolism, vol. 89, no. 6, pp. 2569-2575, 2004.

[11] H. Okubo, S. Sasaki, K. Murakami et al., "Three major dietary patterns are all independently related to the risk of obesity among 3760 Japanese women aged 18-20 years," International Journal of Obesity, vol. 32, no. 3, pp. 541-549, 2008. 
[12] K. Ball and D. Crawford, "An investigation of psychological, social and environmental correlates of obesity and weight gain in young women," International Journal of Obesity, vol. 30, no. 8, pp. 1240-1249, 2006.

[13] H. Takimoto, N. Yoshiike, F. Kaneda, and K. Yoshita, "Thinness among young Japanese women," American Journal of Public Health, vol. 94, no. 9, pp. 1592-1595, 2004.

[14] Health, Labour and Welfare Ministry Home Page, 2003, http:// www.mhlw.go.jp.

[15] S. Tanaka, Y. Itoh, and K. Hattori, "Relationship of body composition to body-fatness estimation in Japanese university students," Obesity Research, vol. 10, no. 7, pp. 590-596, 2002.

[16] E. Stice, E. Schupak-Neuberg, H. E. Shaw, and R. I. Stein, "Relation of media exposure to eating disorder symptomatology: an examination of mediating mechanisms," Journal of Abnormal Psychology, vol. 103, no. 4, pp. 836-840, 1994.

[17] T. Kuboki, S. Nomura, M. Ide, H. Suematsu, and S. Araki, "Epidemiological data on anorexia nervosa in Japan," Psychiatry Research, vol. 62, no. 1, pp. 11-16, 1996.

[18] D. Crawford and K. Ball, "Behavioral determinants of the obesity epidemic," Asia Pacific Journal of Clinical Nutrition, vol. 11, pp. S718-S721, 2002. 


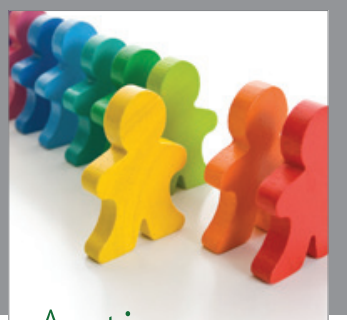

Autism

Research and Treatment
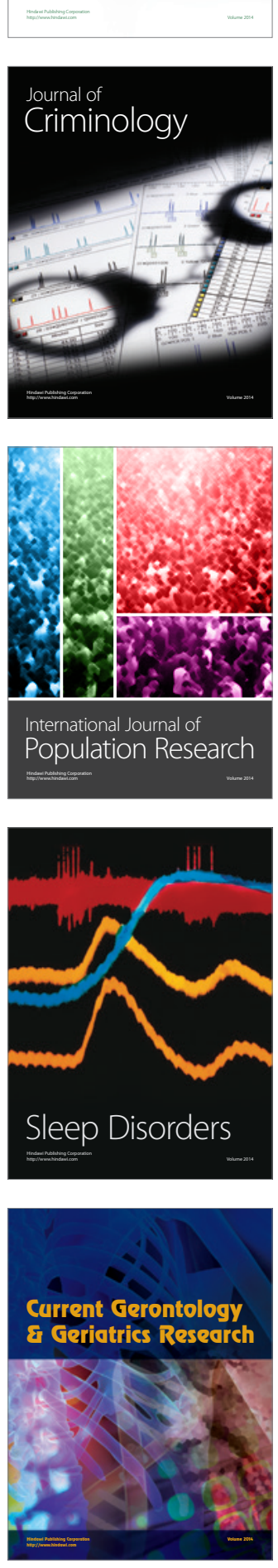
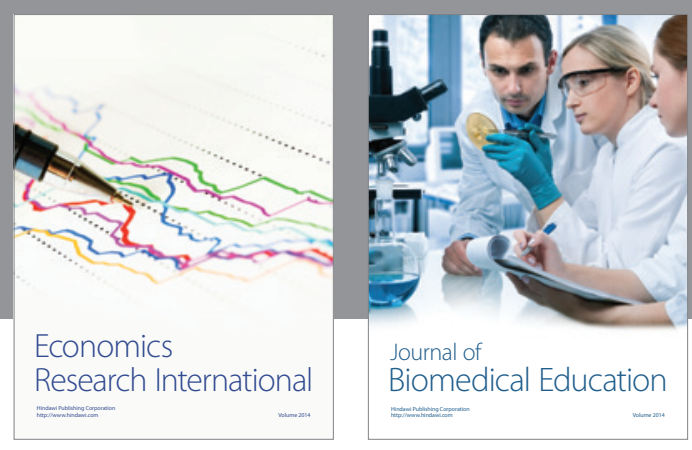

Journal of

Biomedical Education

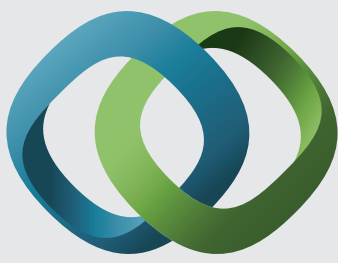

\section{Hindawi}

Submit your manuscripts at

http://www.hindawi.com
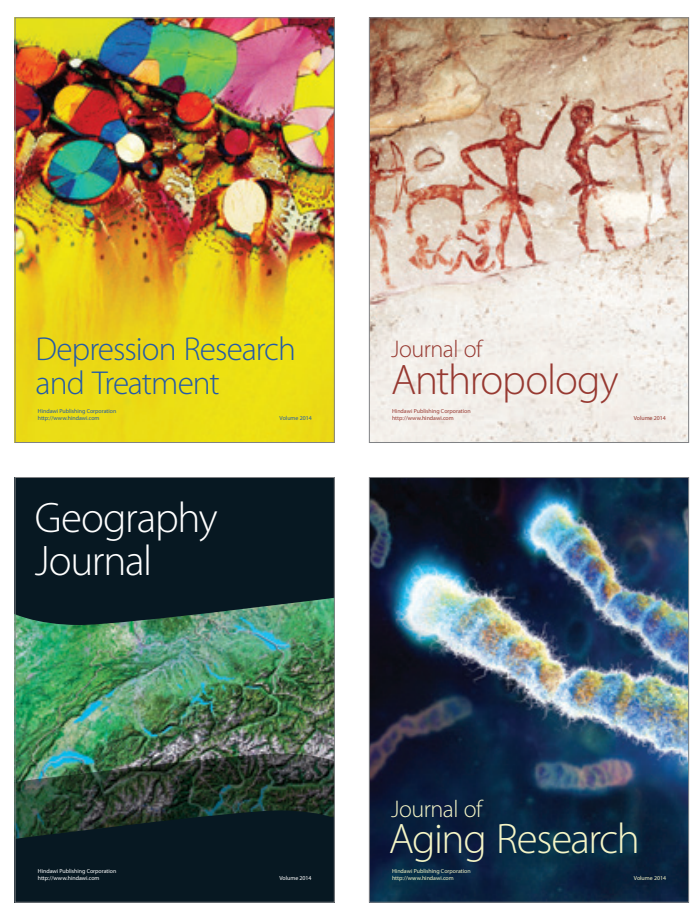

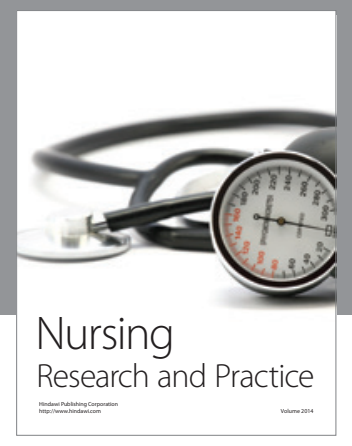

Nursing

Research and Practice

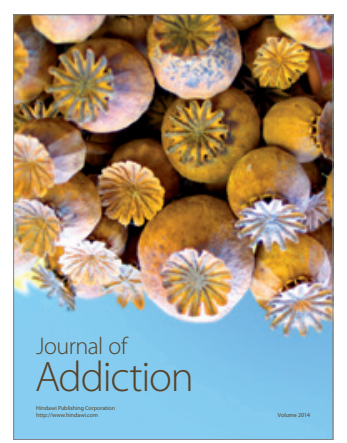

Child Development

Research

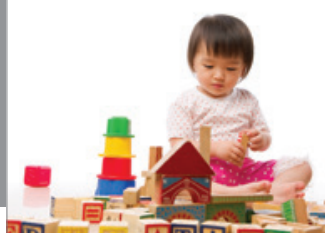

迥
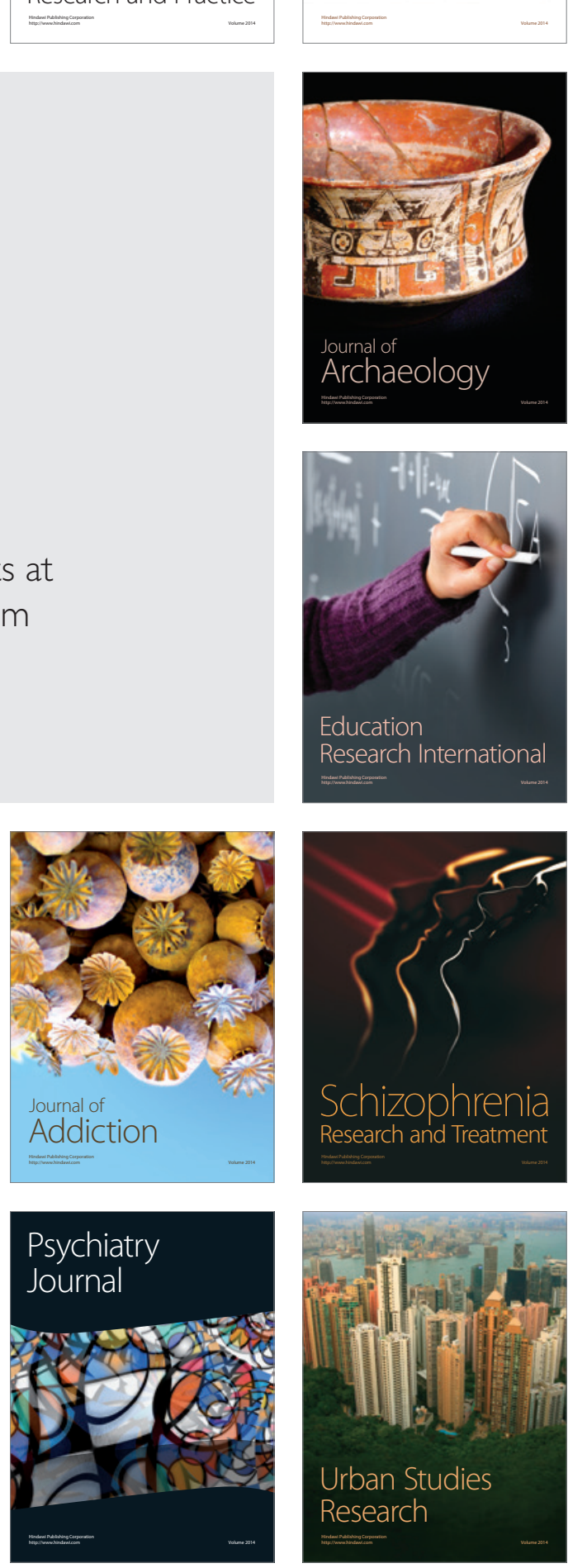